\title{
Optical characterization of high and low resistive silicon samples suitable for reconfigurable antenna design
}

\section{Abdul Ali ${ }^{1}$ (i) I Kagan Topalli $^{2}$ । Mehrab Ramzan ${ }^{3}$ | Talha Masood Khan ${ }^{4}$ । Ayhan Altintas $^{5}$ । Paolo Colantonio ${ }^{1}$}

${ }^{1}$ Department of Electronics Engineering, University of Rome Tor Vergata, Italy

${ }^{2}$ TUBITAK Space Technologies Research Institute, Ankara, Turkey

${ }^{3}$ Chair of RF Engineering, Communication laboratory, TU Dresden, Dresden, Germany

${ }^{4}$ UNAM-Institute of Materials Science and Nanotechnology, Bilkent University, Ankara, Turkey

${ }^{5}$ Department of Electrical and Electronics Engineering and also with the Communication and Spectrum Management Research Center (ISYAM), Bilkent University, Ankara, Turkey

\section{Correspondence}

Abdul Ali, Via Giuseppe Toraldo, 52, 00133, Rome, Italy.

Email: abdul.ali@uniroma2.it

Funding information

H2020 ITN CELTA, Grant/Award Number: 675683

\begin{abstract}
Highly resistive (HR) silicon ( $\mathrm{Si}$ ) can behave as a switch when illuminated by optical source of suitable wavelength. Different reconfigurable passive structures, such as filters, waveguides, and antennas, can be constructed using such silicon switches. This letter presents experimental characterization of high and low resistive (HR \& LR) silicon for switching application. In the experiment, HR Si is modeled on a switched transmission line by halogen lamp and a laser source. The experiment of utilizing halogen lamp for $\mathrm{Si}$ switch characterization is costeffective and can assist engineers in designing reconfigurable antennas. In future, this experiment could be utilized in designing novel reconfigurable antennas.
\end{abstract}

\section{KE YWORDS}

high resistive (HR) silicon, low resistive (LR) silicon, meandered transmission line, optical excitation, halogen lamp, reconfigurable antenna

\section{1 | INTRODUCTION}

Switches are the critical elements in various RF and microwave circuits. Depending on the type of circuit and application, the switch has to satisfy some typical requirements such as low insertion loss, fast switching time, lower DC power consumption, better isolation, and high reliability. When it comes to the design of reconfigurable antenna, the switch should not affect the radiation performance of the antenna ${ }^{1}$ and it should be as compact as possible.

In the past, various switching methods have been used including optical switches, electromagnetic band gap (EBG) structures, PIN diodes, FET switches, varactors, and RF MEMS (micro-electromechanical systems) to design frequency reconfigurable antennas. ${ }^{1-13}$ Among them, optical switches are faster and do not require any biasing circuitry which causes parasitic interference with radiation patterns of the antenna. ${ }^{1,2}$ Typically, their switching time is on the order of nanoseconds.

Nonoptical switching methods, including PIN diodes, FET switches, and varactors, require the design of biasing circuitry which affect the radiation patterns of the antenna. ${ }^{1-12}$ Among them, PIN diodes are preferred for small bias voltages; however, the biasing circuitry increases the complexity of the structure. Additionally, varactors and RF MEMS require large DC bias voltages. The other disadvantages associated with RF MEMS are large switching time, mechanical failure, and low power-handling capability. The EBG structures reported in $^{13}$ require the implementation of six switches. If nonoptical switches are used for their implementation then biasing circuitry for each switch will increase the complexity of the structure.

In this letter, we developed a simple and cost-effective experiment of modeling HR Si for switching applications due to the superior performance of optical switches over nonoptical switches. Silicon (Si) samples with different charge carrier concentration (HR/LR Si) are modeled in a transmission line experiment using halogen lamp and IR laser. A simple circuit model of the switch is contrasted with full EM simulation, and measurement. Experiment with halogen lamp is an alternative solution to expensive laser sources in modeling the optical switches. This experiment and simple circuit model of the switch can help engineers in designing various reconfigurable antennas. 


\section{2 | CHARACTERIZATION OF HR/LR SILICON}

Semiconducting material, such as silicon ( $\mathrm{Si}$ ), is well known for its wide range of interesting properties. Particularly, the optical properties of Si can make it an ideal candidate for switching applications. The conductivity of Si depends on the number of free electrons in the conduction band and holes in the valence band. Thermal energy associated with lattice vibration can create charge carriers (electrons or holes) in $\mathrm{Si}^{14}$ Depending on the band gap energy of $\mathrm{Si}$, additional charge carriers can be created when an optical source of suitable wavelength illuminates the $\mathrm{Si}$ sample. Thus, the overall conductivity of Si sample increases when it is illuminated by an optical source.

Highly resistive (HR) silicon behaves as open (OFF) under no optical illumination. An optical source of suitable wavelength, and power can alter the conductivity of $\mathrm{HR} \mathrm{Si}$ such that it behaves as a short $(\mathrm{ON}) .{ }^{14}$ The conductivity of the Si then depends on the amount of input power from the optical source exciting the sample. Hence, the optical illumination modifies the resistance of the Si sample. On the other hand, LR Si (highly doped) behaves as short (ON) under no optical illumination. At room temperature, large number of charge carriers are created in LR silicon by the thermal energy and electrical conductivity is obtained at this temperature. ${ }^{14}$ Therefore, LR Si is not useful for switching application, whereas the ON state conductivity of HR Si under optical illumination can be compared with LR Si.

In order to observe resistance variation of HR silicon, we choose a very low doped n-type silicon with the resistivity, $\rho$, of $10 \mathrm{k} \Omega . \mathrm{cm}$ and a thickness of $0.5 \mathrm{~mm}$. A recessed $50 \Omega$ transmission line is fabricated and a silicon piece is carefully bonded in the middle section of the transmission line using silver epoxy as shown in Figure 1. The reflection and transmission coefficients of the $50 \Omega$ transmission line are measured using a network analyzer prior to any illumination. Following this, the silicon on the transmission line was illuminated by white light halogen lamp (HL) with diameter, and power density of $50 \mathrm{~mm}$ and $100 \mathrm{~mW} / \mathrm{cm}^{2}$, respectively, and it was powered directly with $220 \mathrm{~V}$ AC. The distance between halogen lamp and Si sample was kept in the range of 10-30 mm. Under HL illumination, we observed some

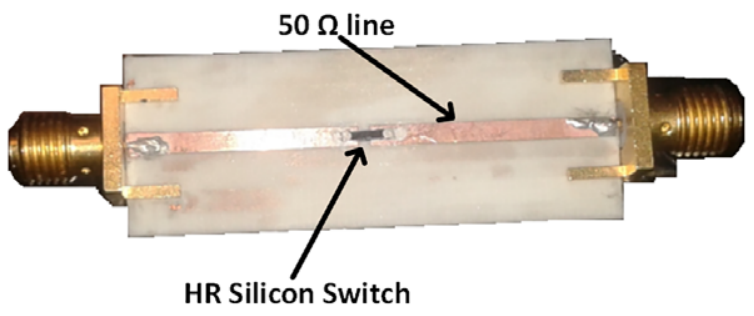

FIGURE $150 \Omega$ transmission line for extracting the impedance of HR Si under halogen lamp illumination [Color figure can be viewed at wileyonlinelibrary.com] variations in the reflection and transmission coefficient of the of the $50 \Omega$ transmission line. If the HL is removed, then, reflection and transmission coefficient of the line move to the original position as was measured prior to any illumination. This initial experiment with HR Si using HL helped in extracting its resistance and capacitance in the OFF and ON state. Table 1 shows the resistance and capacitance of $\mathrm{HR} \mathrm{Si}$ in the two states (ON and OFF). As can be noted, the change in the resistance is quite significant with optical pumping. This remarkable change in resistance of HR Si under HL excitation is enough to bring variations in the surface current redistribution of the antenna. Depending on the geometry of the antenna, the variation in surface current can cause different resonance frequencies. Therefore, the HR Si along with HL can be used to design reconfigurable antennas.

Despite noticeable variation in the resistance under HL illumination, there are two following points that need to be addressed.

1. The ON state resistance of the HR Si in a $50 \Omega$ transmission line experiment is not matched to $50 \Omega$.

2. The intensity of the HL is not precisely controlled.

To address the above points, a meandered transmission line is created in CST Microwave Studio to transform the extracted ON state resistance of HR silicon to $50 \Omega$ under optical illumination at $2 \mathrm{GHz}$. The meandered transmission line is fabricated using RO4003 substrate with a thickness of $0.762 \mathrm{~mm}$ and HR silicon was bonded using silver epoxy. A simple circuit model of the meandered transmission line including HR silicon parameters from Table 1 was also simulated in AWR Microwave Office. The geometrical parameters of the meandered transmission line, fabricated prototype, and circuit model are shown in Figure 2.

The HL experiment is repeated with meandered transmission line containing HR Si to address the improvement in matching. The experimental results obtained are depicted in Figures 3 and 4 for the OFF (without illumination) and ON (with illumination) state, respectively. As can be seen from the plots that the measured results are generally in good agreement with the simulations and circuit model.

In the OFF state, the silicon exhibits a very high impedance $\left(R_{x}=15.8 \mathrm{k} \Omega\right)$ and it is not matched to $50 \Omega$. Therefore, in the OFF state, almost no power is transferred from port 1 to port 2 . In the $\mathrm{ON}$ state, the reflection coefficient is close to $-10 \mathrm{~dB}$ and the matching to $50 \Omega$ port is reasonable. However, the transmission coefficient is slightly changed. The reason for this slight shift is the power from the HL

TABLE 1 Circuit model parameters of HR silicon extracted from halogen lamp experiment

\begin{tabular}{lll} 
State & ${ }^{\mathbf{R}}$ & $\mathbf{C}_{\mathbf{x}}$ \\
\hline OFF & $15.8 \mathrm{k} \Omega$ & $47 \mathrm{fF}$ \\
ON & $512 \Omega$ & $67 \mathrm{fF}$ \\
\hline
\end{tabular}




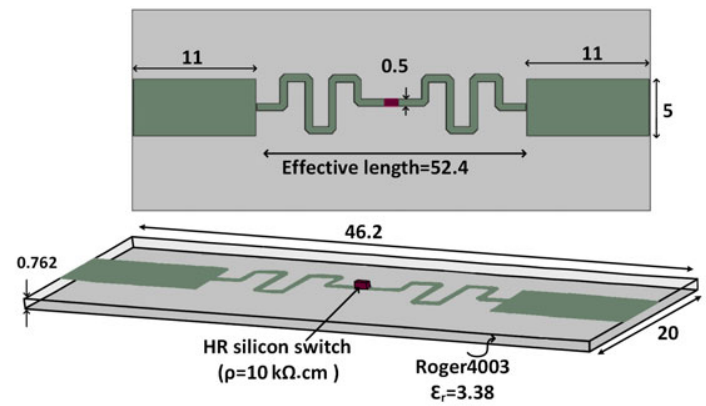

(A)

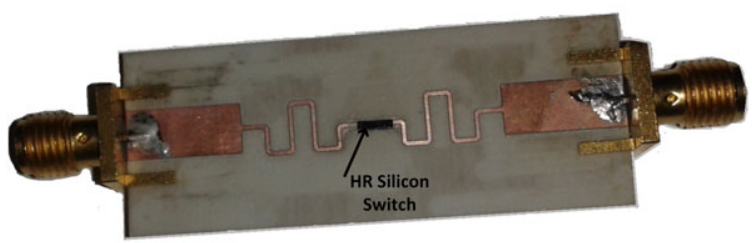

(B)

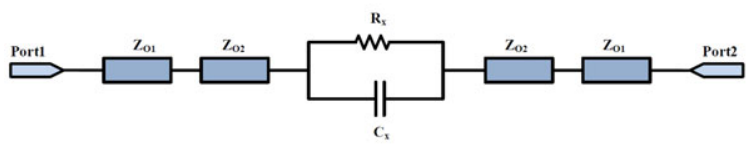

(C)

FIGURE 2 Meandered transmission line to transform the impedance of HR/LR Si $50 \Omega$. geometry of meandered transmission line used in simulation (A), fabricated prototype (B) and equivalent circuit model (C) [Color figure can be viewed at wileyonlinelibrary.com]

is not concentrated toward a particular direction. A more concentrated power on the silicon switch is required. To control the intensity of optical source, the experiment with meandered transmission line is repeated with a laser source (IR laser, $\lambda=1035 \mathrm{~nm}$ ). The spot size of the laser beam was on the order of $1 \mathrm{~mm}$ in diameter. The laser source was placed at $500 \mathrm{~mm}$ away from the circuit. The power from laser source is varied and the corresponding reflection

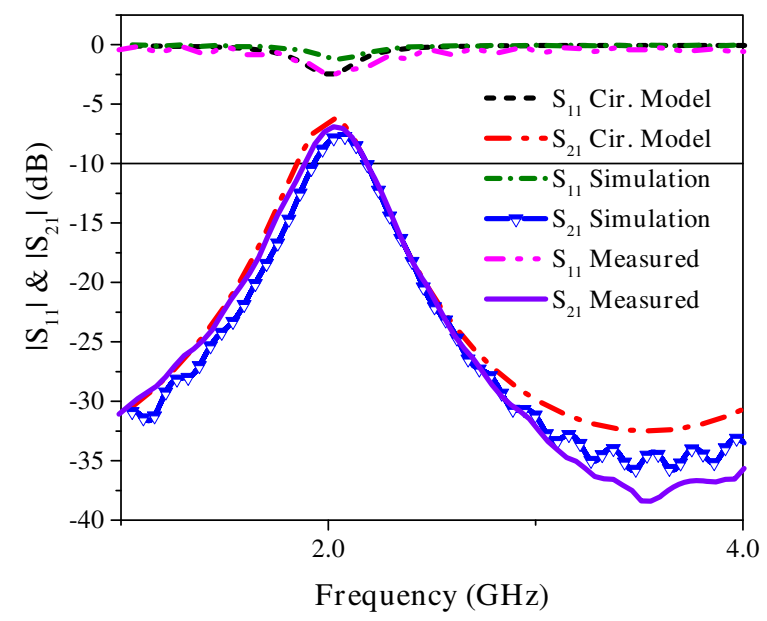

FIGURE 3 Scattering parameters of meandered transmission line in the OFF state. The line type show S-parameters obtained from circuit model, EM simulation, and measurement [Color figure can be viewed at wileyonlinelibrary.com]

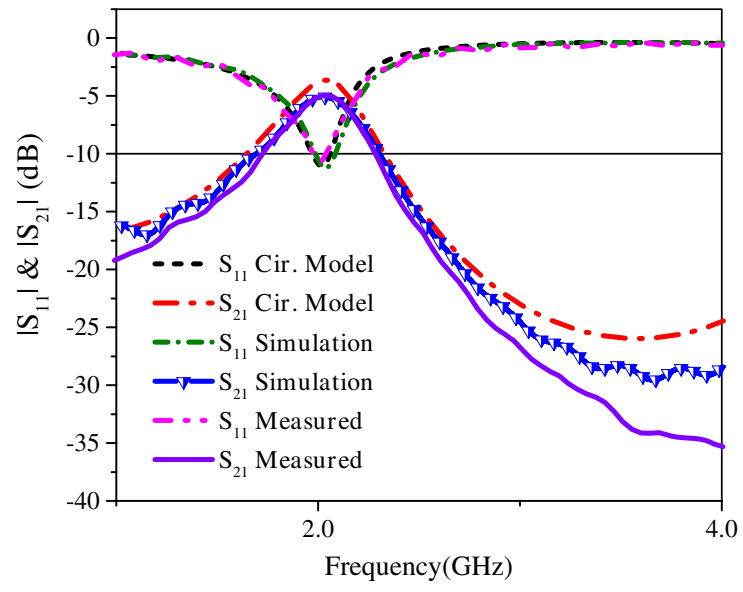

FIGURE 4 Scattering parameters of meandered transmission line in the ON state. The line type show S-parameters obtained from circuit model, EM simulation, and measurement [Color figure can be viewed at wileyonlinelibrary.com]

coefficient is measured. In addition, LR Si (highly doped) with resistivity, $\rho$, of $1-10 \Omega . \mathrm{cm}$ is bonded to meandered transmission line using silver epoxy.

The S-parameters of the LR (at room temperature) and HR Si under HL and laser excitation are reported in Figure 5. A low power level of $7.5 \mathrm{~mW}$ from IR laser source makes the HR Si sufficiently conductive which is equivalent to the conductivity achieved with HL excitation. At $50 \mathrm{~mW}$ of power, the HR $\mathrm{Si}$ becomes highly conductive and it results in improvement of Sn and S21. Evidently, it can be noted that HR Si with power $\geq 50 \mathrm{~mW}$ illumination resulted in almost similar conductivity attained with LR Si. Therefore, a power $\geq 50 \mathrm{~mW}$ from IR laser is enough to make HR Si conductive.

\section{3 | CONCLUSIONS}

This letter presented experimental characterization of HR and LR Si for switching application using different optical

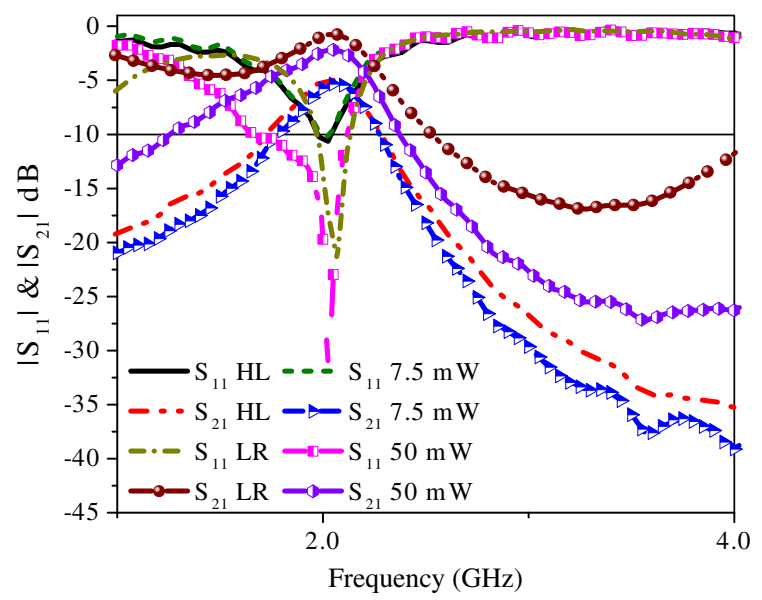

FIGURE 5 Comparison of measured scattering parameters of meandered transmission line containing LR and HR Si. The HR Si is illuminated by a halogen lamp (HL) and IR laser with different power level [Color figure can be viewed at wileyonlinelibrary.com] 
sources. Dramatic variations in the resistance of HR Si under HL illumination are observed. Such prominent variations in the resistance of HR Si are highly desirable for reconfigurable antenna design. The experiment with HL is costeffective and easy to interface. The simplified circuit model of $\mathrm{HR} \mathrm{Si}$ and additional characterization with IR laser can further assist in designing various reconfigurable passive circuits.

This letter also characterizes LR Si in a transmission line experiment. The LR Si in order to imitate the switch under illumination can be a viable solution to conduct radiation pattern measurement of reconfigurable antennas without the need of optical source and fiberoptic cable in the anechoic chamber. In future, this experiment can be utilized to design various optically reconfigurable antennas for different applications.

\section{ACKNOWLEDGMENT}

The authors thank the H2020 ITN CELTA under grant number 675683 of Call: H2020-MSCA-ITN-2015.

\section{ORCID}

Abdul Ali (D) https://orcid.org/0000-0002-0095-6702

\section{REFERENCES}

[1] Panagamuwa CJ, Chauraya A, Vardaxoglou JC. Frequency and beam reconfigurable antenna using photoconducting switches. IEEE Trans Antennas Propag. 2006;54(2):449454.

[2] Tawk Y, Albrecht AR, Hemmady S, Balakrishnan G, Christodoulou CG. Optically pumped frequency reconfigurable antenna design. IEEE Antennas Wirel Propag Lett. 2010;9: 280-283.

[3] Silva LG, Alves AAC, Sodre AC. Optically controlled reconfigurable filtenna. Int J Antennas Propag. 2016;2016:1-9.

[4] Collett MA, Gamlath CD, Cryan MJ. Optically induced conductivity in silicon: an active control technique for antennas. Microw Opt Technol Lett. 2016;58(4):994-998. [Online]. Available: https://onlinelibrary.wiley.com/doi/abs/10.1002/mop.29713
[5] Pendharker S, Shevgaonkar RK, Chandorkar AN. Optically controlled frequency-reconfigurable microstrip antenna with low photoconductivity. IEEE Antennas Wirel Propag Lett. 2014;13: 99-102.

[6] Tawk Y, Costantine J, Hemmady S, Balakrishnan G, Avery K, Christodoulou CG. Demonstration of a cognitive radio front end using an optically pumped reconfigurable antenna system (OPRAS). IEEE Trans Antennas Propag. 2012;60(2):10751083.

[7] Nikolaou S, Bairavasubramanian R, Lugo C, et al. Pattern and frequency reconfigurable annular slot antenna using PIN diodes. IEEE Trans Antennas Propag. 2006;54(2):439-448.

[8] Pringle LN, Harms PH, Blalock SP, et al. A reconfigurable aperture antenna based on switched links between electrically small metallic patches. IEEE Trans Antennas Propag. 2004;52(6):1434-1445.

[9] Tawk Y, Costantine J, Christodoulou CG. A varactor-based reconfigurable filtenna. IEEE Antennas Wirel Propag Lett. 2012; 11:716-719.

[10] Tariq A, Ghafouri-Shiraz H. Frequency-reconfigurable monopole antennas. IEEE Trans Antennas Propag. 2012;60(1):44-50.

[11] Chiu CY, Li J, Song S, Murch RD. Frequency-reconfigurable pixel slot antenna. IEEE Trans Antennas Propag. 2012;60(10): 4921-4924.

[12] Erdil E, Topalli K, Unlu M, Civi OA, Akin T. Frequency tunable microstrip patch antenna using RF MEMS technology. IEEE Trans Antennas Propag. 2007;55(4):11931196.

[13] Majid HA, Kamal M, Rahim A, et al. Reconfigurable notched wideband antenna using EBG structure. Microw Opt Technol Lett. 2015;57(2):497-501. [Online]. Available: https://onlinelibrary. wiley.com/doi/abs/10.1002/mop.28882

[14] Callister WD Jr, Rethwisch DG. Materials Science and Engineering: An Introduction. 9th ed. New York: Wiley; 2013.

How to cite this article: Ali A, Topalli K, Ramzan M, Khan TM, Altintas A, Colantonio P. Optical characterization of high and low resistive silicon samples suitable for reconfigurable antenna design. Microw Opt Technol Lett. 2019;61:107-110. https:// doi.org/10.1002/mop.31506 